\title{
Squeezed-state generation in optical bistability
}

\author{
L. A. Orozco, M. G. Raizen, Min Xiao, R. J. Brecha, and H. J. Kimble \\ Department of Physics, University of Texas at Austin, Austin, Texas 78712
}

Received May 18, 1987; accepted June 25, 1987

\begin{abstract}
Experiments to generate squeezed states of light are described for a collection of two-level atoms within a highfinesse cavity. The investigation is conducted in a regime for which the weak-field coupling of atoms to the cavity mode produces a splitting in the normal mode structure of the atom-field system that is large compared with the atomic linewidth. Reductions in photocurrent noise of $30 \%(-1.55 \mathrm{~dB})$ below the noise level set by the vacuum state of the field are observed in a balanced homodyne detector. A degree of squeezing of approximately $50 \%$ is inferred for the field state in the absence of propagation and detection losses. The observed spectrum of squeezing extends over a very broad range of frequencies $(\sim \pm 75 \mathrm{MHz})$, with the frequency of best squeezing corresponding to an offset from the optical carrier given by the normal mode splitting.
\end{abstract}

\section{INTRODUCTION}

The nonlinear-optical interactions considered for squeezedstate generation are often too weak to generate significant degrees of squeezing over a single Rayleigh length. To remedy this situation optical cavities can be employed to enhance the effective nonlinearity with multiple passes through the medium. A balance of gain and loss then leads to threshold conditions near which large degrees of squeezing can be generated. ${ }^{1}$ In optical physics such cavity configurations are usually operated in a regime for which the relaxation rate of the intracavity medium is large compared with the cavity decay rate. The intracavity medium then adiabatically follows variations in the cavity field and can be considered to be in steady state. This is the so-called goodcavity limit of laser physics and of optical bistability and is the limit applicable to recent squeezed-state experiments ${ }^{2,3}$ and theories. ${ }^{4-6}$

By returning to the problem of squeezed-state generation in optical bistability ${ }^{4,5}$ and by exploring the full dynamic problem of the coupled atom-field dynamics, we have identified a new regime for the generation of squeezed states of the electromagnetic field.7,8 The coupling of a collection of $N$ two-level atoms to a single mode of a cavity introduces a new structure not found in the physics of the intracavity medium or of the cavity mode taken separately. For a weak intracavity field and zero detunings, the normal mode structure is characterized by a frequency known as the vacuumfield Rabi splitting, ${ }^{9-12}$ which is just a normal mode splitting of magnitude $g \sqrt{N}$ in the eigenmode structure of the composite atom-field system. Here $g$ is the single-atom coupling coefficient (single-photon Rabi frequency), and $N$ is the number of atoms in the interaction volume. In the microwave domain, the normal mode structure was observed in experiments with Rydberg atoms ${ }^{13,14}$ down to the scale of a single atom $(N=1),{ }^{14}$ and temporal squeezing in a Rydberg atom maser was proposed. ${ }^{15}$ Unfortunately, in the optical domain the frequency $\Omega_{0} \equiv g \sqrt{N}$ is usually overshadowed by the large damping rates of the cavity field $(\kappa)$ and of the atomic polarization $\left(\gamma_{\perp}\right)$ and inversion $(\gamma)$. To understand this point, note that, in the presence of dissipation, Carmichae ${ }^{11}$ has shown that the eigenvalues of the linearized system in the weak field limit (for which the atoms are nearly always in the ground state) are $\lambda_{ \pm}=-(1 /$ 2) $(\gamma / 2+\kappa) \pm\left[(1 / 4)(\gamma / 2-\kappa)^{2}-g^{2} N\right]^{1 / 2}$, where zero detunings and radiative damping $2 \gamma_{\perp}=\gamma$ are assumed. We see that $\lambda_{ \pm}$contains an imaginary part only for $1 / 2\left|\gamma / 2-{ }_{k}\right|<$ $g \sqrt{N}$. That is, a periodic exchange of excitation occurs only if the difference of decay rates of the two oscillators is smaller than the coupling frequency. Although there are exceptions, it is usually the case in optical physics that the decay rates $\left(\kappa, \gamma_{\perp}, \gamma\right)$ are dissimilar and the coupling frequency $g \sqrt{N}$ is small, so the splitting that we have described is not observed. It is possible, however, to design a system at optical wavelengths for which $g \sqrt{N} \gg\left(\kappa, \gamma_{\perp}, \gamma\right)$. Indeed, we probed the coupling-induced structure for a collection of two-level atoms in a cavity by monitoring the transient response to step excitation. We observe the oscillatory exchange of excitation between two oscillators-the collective atomic polarization and the cavity mode. For weak intracavity fields, the frequency of oscillation is observed to be the coupling rate $g \sqrt{N}$ characteristic of the normal mode structure of the coupled oscillators. ${ }^{16}$

The presence of this dynamic process suggests its possible use for the generation of squeezed states. In qualitative terms, we wish to inquire whether the eigenmode structure of the composite system can lead to phase-sensitive amplification and deamplification of quantum fluctuations. We shall see that significant degrees of squeezing can, in fact, be generated and that the spectrum of squeezing is peaked around the coupling frequency $g \sqrt{N}$. This situation is contrary to the literature on squeezed-state generation by four-wave mixing that predicts squeezing at frequency offsets from the carrier characterized by the generalized Rabi frequency for strong intracavity intensity.

The principal focus of this paper is a detailed discussion of the experiments that we have conducted to generate squeezed states by the mechanism discussed above. Our system consists of a driven optical cavity whose axis intersects at $90^{\circ}$ an atomic beam of optically prepumped sodium atoms. ${ }^{17}$ The experiment can be modeled to a good approximation as a collection of two-level atoms interacting with a single mode of an optical cavity. ${ }^{18}$ Dissipation and damping enter predominantly through the radiative decay 
of the atoms and through the transmission of the cavity field by a mirror.

We observed squeezing in two different cavity and atomic beam arrangements with quite different coupling coefficients. In each case we have recorded noise reductions in a balanced homodyne detector below the level set by the vacuum state of the field. In our most recent experiments, noise reductions of $30 \%(-1.55 \mathrm{~dB})$ were observed. The frequency at which best squeezing occurs in both cavity configurations is approximately that of the coupling coefficient $g \sqrt{N}$ and is in good agreement with our theoretical calculations, which are based on previously presented quantum-statistical theories of optical bistability. ${ }^{11,19-23}$

The organization of the paper is as follows. In Section 2 we present a brief summary of our theoretical analysis. Section 3 is devoted to a detailed discussion of the experimental apparatus. Section 4 describes the alignment and operation of the system. In Section 5 we present our observations of squeezed-state generation. Section 6 serves as a conclusion.

\section{THEORETICAL OVERVIEW}

We begin with an analysis of the interaction of a collection of $N$ two-level atoms with a single mode of a high-finesse interferometer. The model Hamiltonian $\hat{H}$ for this system has been extensively studied in quantum optics and is taken to be of the form ${ }^{19-23}$

$$
\begin{aligned}
\hat{H} & =\hat{H}_{0}+\hat{H}_{a}+\hat{H}_{c}, \\
\hat{H}_{0} & =\left(\hbar \omega_{a} / 2\right) \hat{J}_{z}+\left(\hbar \omega_{c}\right) \hat{a}^{\dagger} \hat{a}+\hbar g\left[i \hat{J}_{-} \hat{a}^{\dagger}+\text { H.C. }\right],
\end{aligned}
$$

where Hilbert space operators are denoted by a caret .

The coherent coupling of the atomic polarization to the cavity field is described by $\hat{H}_{0} . \quad\left(\hat{J}_{z}, \hat{J}_{ \pm}\right)$are collective atomic operators for the $N$ atoms of transition frequency $\omega_{a}$ and $(\hat{a}$, $\left.\hat{a}^{\dagger}\right)$ are the annihilation and creation operators for the single cavity mode of resonant frequency $\omega_{c}$. The atoms and field modes are coupled through an assumed dipole interaction with coupling coefficient $g=\left(\omega_{c} \mu_{0}^{2} / 2 \hbar \epsilon_{0} V\right)^{1 / 2}$, where $\mu_{0}$ is the dipole moment and $V$ the cavity-mode volume. Decay of the atomic inversion is assumed to be purely radiative at rate $\gamma$, while the atomic polarization decay rate is designated by $\gamma_{\perp}$. Both decay processes are described by $\hat{H}_{a}$. The field amplitude decays at a rate $\kappa$ by coupling through the cavity mirrors to a set of continuum input-output modes, as described by $\hat{H}_{c}$, which also includes the possibility of excitation by an external field of frequency $\omega_{1}$.

Of the diverse quantum-statistical properties of the Hamiltonian [Eq. (1)], we wish to focus on the particular problem of optical bistability for which equations of motion for generalized quasi-probability distributions were previously derived in the literature. ${ }^{19,21,23}$ In particular, we consider the generalized Fokker-Planck equation in the positive $P$ representation without adiabatic elimination of atomic or field variables. ${ }^{12,21}$ This equation is linearized about the steady-state solution, and the resulting equation is solved for the spectral density $A(\phi, \Omega)$ that describes the fluctuations of the quadrature amplitude $\hat{z}(t)$ of the intracavity field, where $\hat{z}(t)=\hat{a}(t) e^{-i \phi}+\hat{a}^{\ddagger}(t) e^{i \phi}$ and

$$
A(\phi, \Omega)=\int_{-\infty}^{\infty} \mathrm{d} \tau e^{-i \Omega \tau}\langle: \hat{z}(t, \phi), \hat{z}(t+\tau, \phi):\rangle .
$$

In this equation, the covariance $\langle\hat{a}, \hat{b}\rangle \equiv\langle\hat{a} \hat{b}\rangle-\langle\hat{a}\rangle\langle\hat{b}\rangle$, the colons denote time and normal ordering (with annihilation operators in ascending time order from the right and creation operators in ascending time order from the left), and the time $t$ is sufficiently long to ensure a (stationary) steady state. Squeezing of the field emitted through the output mirror of the cavity is described by the spectrum of the squeezing $S(\phi, \Omega)$, where ${ }^{24,25}$

$$
S(\phi, \Omega)=2 \kappa A(\phi, \Omega) .
$$

A detailed discussion of our theoretical analysis will be presented in a separate publication. ${ }^{26}$ For the moment, note that $S(\phi, \Omega)$ is defined such that $S=0$ for either a coherent state or a vacuum state, while $S_{-}(\Omega) \equiv S\left(\phi_{-}, \Omega\right) \rightarrow$ -1 and $S_{+}(\Omega) \equiv\left(\phi_{-}+\pi / 2, \Omega\right) \rightarrow \infty$ for perfect squeezing. The angle $\phi_{-}$defines the quadrature of optimum squeezing and depends on the frequency $\Omega$ for the processes considered here.

Our results for the spectrum of squeezing are displayed in Fig. 1 for several sets of operating conditions relevant to the experiments described in Sections 3-5. The external control parameters are the atomic detuning $\Delta \equiv\left(\omega_{a}-\omega_{1}\right) / \gamma_{\perp}$, the cavity detuning $\theta \equiv\left(\omega_{\mathrm{c}}-\omega_{1}\right) / \kappa$, the atomic cooperativity parameter $C=N g^{2} / \gamma \kappa$, the intracavity field $x=\langle\hat{a}\rangle / \sqrt{n}_{0}$, with $n_{0}=\gamma_{\perp} \gamma / 4 g^{2}$, and the ratio of cavity losses to atomic decay rate $\mu=\kappa / \gamma$. A dimensionless frequency $v=\Omega /(g \sqrt{N})$ is introduced as the abcissa in the figure. Note that the frequency $v=1$ corresponds to the mode splitting described in Section 1 and that the regions of optimum squeezing are centered near these values, even though the parameter sets considered in Fig. 1 differ substantially. While the width of the squeezing may appear to be fairly narrow in Fig. 1, the actual frequency range can be quite large. For example, for curve (iii) in the case of sodium vapor, the full width of the region over which $S_{-}<-0.50$ is approximately 670 $\mathrm{MHz}$.

A more global picture of the nature of squeezing in this system is obtained from Fig. 2, which shows the dependence of $S_{-}$on atomic cooperativity parameter $C$. In Fig. 2 each point results from a search for optimum squeezing over all parameters $(\Delta, \theta, \Omega, x)$ for given values of $(C, \mu)$. This

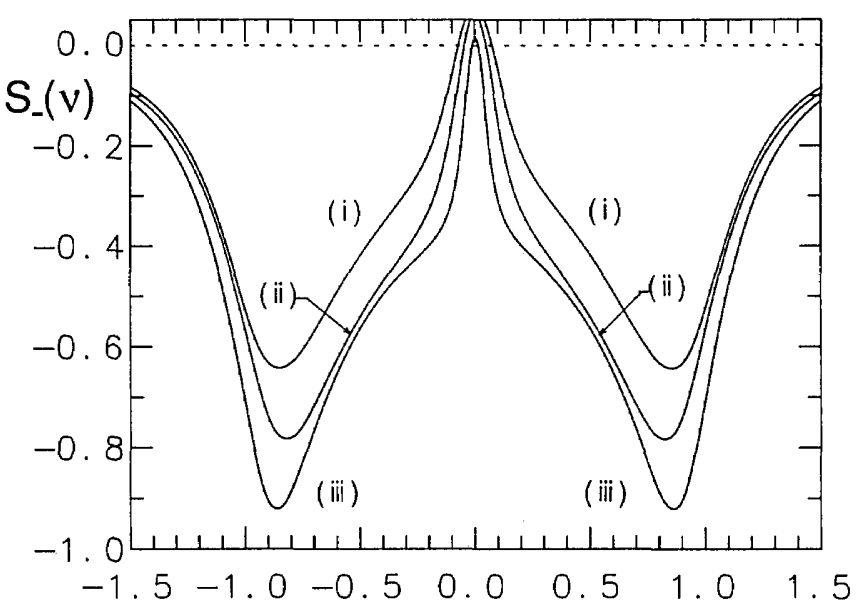

Fig. 1. Spectrum of squeezing $S_{-}$versus offset frequency $\nu=\Omega /$ $g \sqrt{N}$, with $g \sqrt{N}=\gamma \sqrt{\mu} C$. Trace (i) $\mu=5.4, C=28, \Delta=7.3, \theta=-0.9, x$ $=7.0$; trace (ii) $\mu=13.5, C=52, \Delta=-14.6, \theta=0.86, x=16.4$; trace (iii) $\mu=50, C=200, \Delta=60.0, \theta=-1.0, x=70$. 


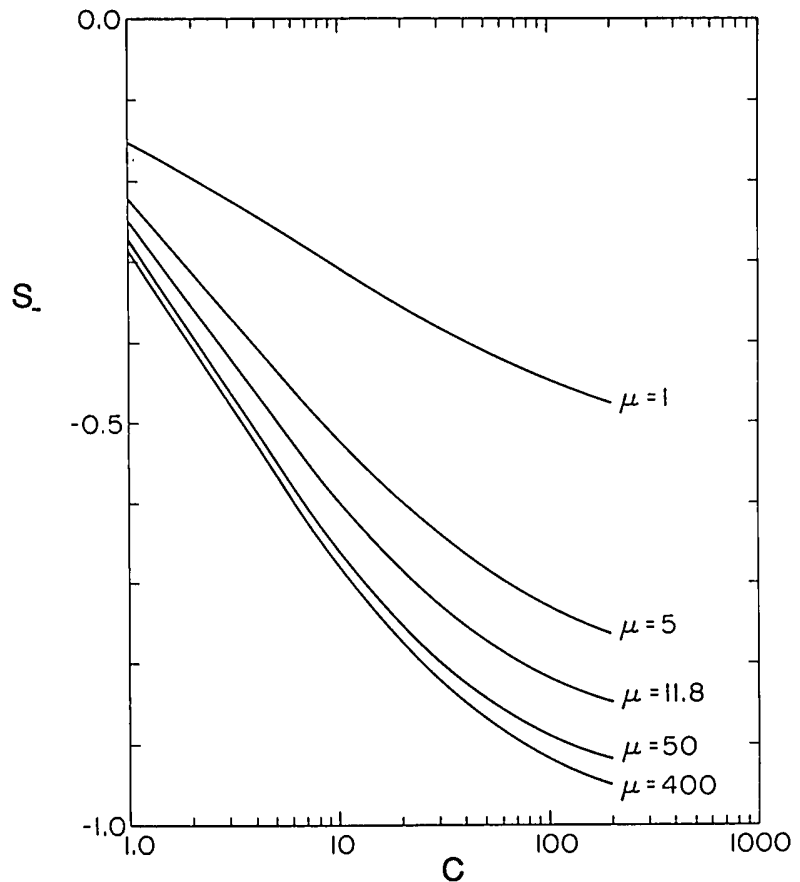

Fig. 2. Dependence of the minimum value of the spectrum of squeezing $S_{-}$on atomic cooperativity $C$ for fixed $\mu$. Note that, at any point on a curve, the values of $(\Delta, \theta, \Omega, x)$ were chosen for optimum squeezing.

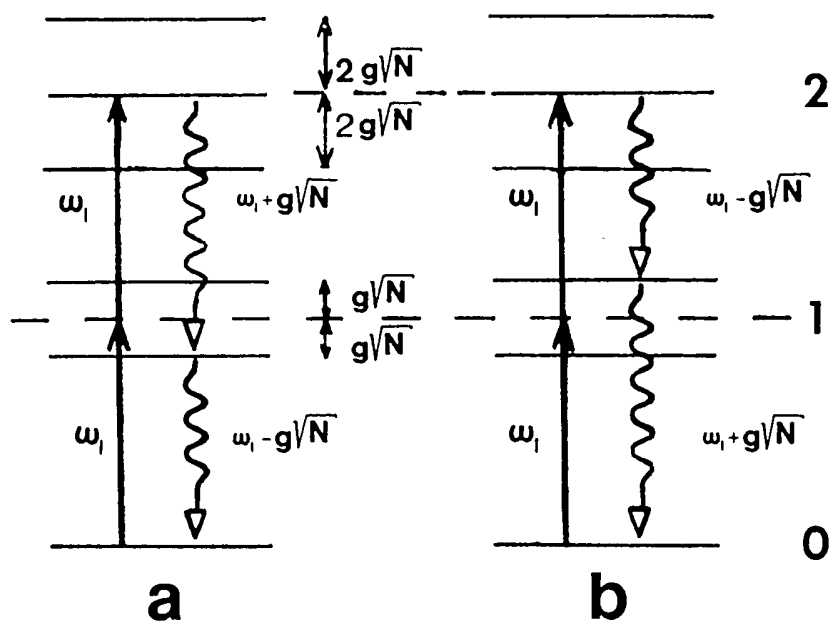

Fig. 3. Diagram of dressed states for $N \gg 1$ atoms coupled to a single mode of a cavity. The three groups of states $(0,1,2)$ correspond to zero, one and two excitations in the system. The degeneracy of the noninteracting system is lifted, in the presence of coupling, to produce splittings characterized by the frequency $g \sqrt{N}$. The figure is drawn for $\Delta=\theta=0$. The solid lines represent absorption of coherent pump photons, while the wavy lines in a and b depict two possible return paths to the ground state (following Varada et $\left.a l .{ }^{28}\right)$.

search is carried out in four-dimensional space with the value of $S_{-}$at any given point in the space requiring the inversion of five-dimensional matrices. Note that substantial degrees of squeezing are predicted for relatively modest values of $(\mathrm{C}, \mu),{ }^{7-9,27}$ relative to earlier treatments of squeezed-state generation with two-level atoms. ${ }^{4-6}$ In addition to the results presented in Figs. 1 and 2, we also constructed isosqueezing contours that indicate that squeezing in this new regime persists over relatively broad regions of the parameter space so that, in practice, the predicted effects should be reasonably robust with respect to deviations between actual experiment and the model calculations.

As a final qualitative description of our results for squeezed-state generation, we consider the level diagram in Fig. 3. This figure is taken from the work of Varada et al. ${ }^{28}$ and is a schematic diagram of the first few dressed states for a system of $N \gg 1$ atoms interacting with the cavity field. For squeezed-state generation, we consider fourwave mixing among these low-lying ( $N$-atom) + (cavitymode) states. For example, the solid lines in the figure represent the absorption of two pump photons at frequency $\omega_{1}$ from the coherent intracavity field followed by the emission of a correlated pair of signal and idler photons at the frequencies $\omega_{1} \pm g \sqrt{N}$. Two possible emission sequences are shown in Figs. 3a and 3b. Although Fig. 3 is drawn for zero atomic and cavity detunings, optimum squeezing occurs for nonzero $(\Delta)$. The dressed-state structure of Fig. 3 and the corresponding eigenvalues of the Maxwell-Bloch equation are then considerably more complex. ${ }^{26,28}$ However, the essential features of the foregoing discussion remain unchanged. We should stress that we did not pursue a quantitative analysis based on Fig. 3; the figure is offered in an attempt to make contact with the literature in nondegenerate four-wave mixing.

From this brief discussion we attempt to distill the essential ingredients for the generation of squeezed states. First, the effective coupling coefficient $g \sqrt{N}=\gamma \sqrt{\mu C}$ must be sufficiently large relative to the damping rates $(\kappa, \gamma)$ to ensure a splitting. The cavity decay rate $\kappa$ should be much larger than the atomic decay rate $\gamma$ so that the dominant decay route for the spectral features of $A(\Omega)$ is through the cavity output coupler. With these conditions satisfied, the intracavity field needs to be increased to the point $x \sim \Delta$ to provide sufficient excitation for the mixing processes depicted in Fig. 3.

As a final note, we emphasize that either in the good- or bad-cavity limits of optical bistability, for which either the atomic $(\mu \rightarrow 0)$ or the cavity $(\mu \rightarrow \infty)$ dynamics are adiabatically eliminated, the structure that we identified vanishes, as does the squeezing generated from it. The specification $\mu \rightarrow 0$ or $\mu \rightarrow \infty$ for fixed $\mathrm{C}$ is not sufficient to justify adiabatic elimination of either atomic or field variables; the magnitude of the coupling frequency $g \sqrt{N}$ as well as that of the detunings $(\Delta, \theta)$ must also be considered. For example, in Eq. (5) of Ref. 6 for which the good-cavity limit was assumed, the eigenvalue of the drift matrix [Eq. (26) of Ref. $6]$ is $\bar{\lambda}=-\mu[1+2 C /(1+i \Delta)]$ to zeroth order in the pump field. This is precisely the same result obtained for the eigenvalues in our case (which are $\lambda^{ \pm}$given here for no detunings) in the limit $\mu \rightarrow 0$ and the weak field. We thus see that in the usual good-cavity limit the imaginary component of the eigenvalue given by $g \sqrt{N}$ is lost. Squeezing can, nonetheless, be generated in either the good- or bad-cavity limits, but by different avenues from those associated with the structure that we have identified and which is illustrated in Fig. 3.

\section{EXPERIMENTAL APPARATUS}

In this and the following section we describe in detail our experimental apparatus. The system is designed to be a reasonable realization of the model system described by the 
Hamiltonian of Eqs. (1). Figure 4 is a diagram of our apparatus. A frequency-stabilized ring dye laser (Coherent 69921) (laser 1 ) is the principal source of excitation. Part of this laser is split off at the beam splitter (BS1) to serve as the local oscillator (LO), while the other beam from BS1 (signal beam) drives the cavity for squeezed-state generation. The signal beam can be intensity modulated at high frequency by the electro-optic modulator (EO1) or frequency shifted by an acousto-optic modulator (AO1). Low-frequency control of the driving intensity is obtained by a half-wave plate ( $\mathrm{H} 1)$ between crossed polarizers (P1, P2) and with electro-optic modulator EO2 and polarizer P3. A constant fraction of the

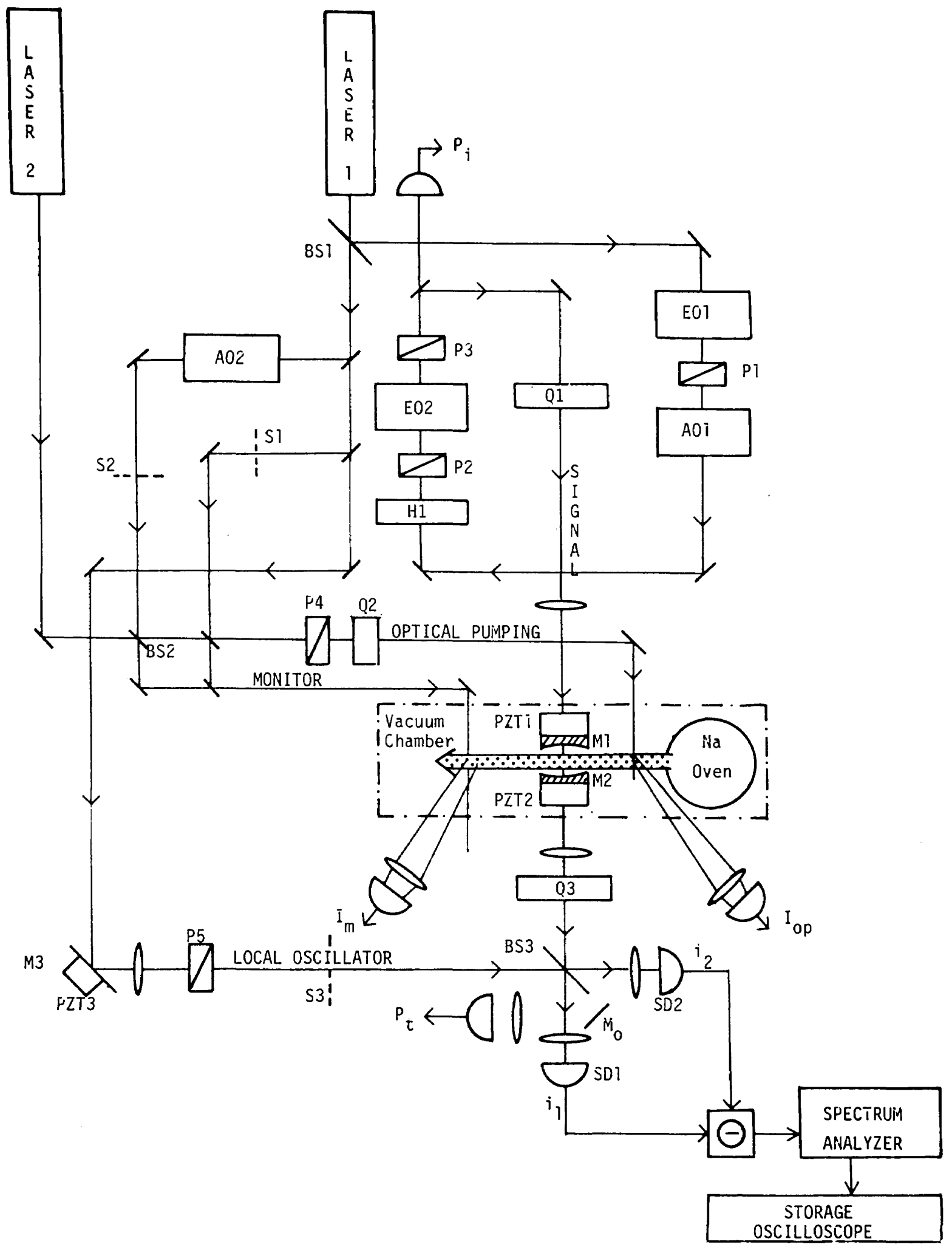

Fig. 4. Diagram of the elements of the experiment for generating and detecting squeezed states. BS1, BS2, BS3, beam splitters; EO1, EO2, electro-optic modulators; P1, P2, P3, P4, P5, polarizers; AO1, AO2, acousto-optic modulators; Q1, Q2, Q3, quarter-wave plates; H1, half-wave plate; S1, S2, S3, shutters; M1, M2, M3, mirrors; SD1, SD2, photodiodes; PZT1-PZT3, piezoelectric transducers. 
power from $\mathrm{P} 3$ is directed onto a photodiode $\left(\mathrm{P}_{i}\right)$ calibrated with respect to the actual power incident upon the squeezing cavity formed by mirrors M1 and M2. The polarization of the signal beam is converted to circularly polarized light with the quarter-wave plate (Q1). The signal beam is mode matched to the squeezing cavity with an efficiency of approximately $90 \%$.

Several laser beams in addition to the signal and LO beams are employed in the experiments. Two beams are split from the LO beam to prepump the atomic sodium optically into the $3^{2} S_{1 / 2}, F=2, m_{F}=2$ state of the $D_{2}$ line $(589 \mathrm{~nm})$. One of these beams is frequency shifted with acousto-optic modulator $\mathrm{AO} 2$ to permit a fixed detuning between the optical pumping and the signal beam. The other pumping beam is collinear with the frequency-shifted one and is used for initial alignment and for measurements of the input-output characteristics in absorptive bistability. Before interacting with the atoms, the polarizations of all the optical pumping beams are converted to circular polarization with polarizer P4 and quarter-wave plate Q2. Shutters $\mathrm{S} 1$ and $\mathrm{S} 2$ allow one or both of the beams to be blocked. A second dye laser (Coherent 599-21) (laser 2) injected through BS2 transfers the population from the $F=1$ to the $F$ $=2$ ground state by the $F=2$ excited state to increase the optical density of the atomic beam entering the squeezing cavity.

In most of our experiments, the fluorescence from the optical pumping beam $\left(I_{o p}\right)$ is used to lock the frequency of laser 1. The pumping beam is offset by $-73 \mathrm{MHz}$ by the acousto-optic modulator $\mathrm{AO} 2$ relative to the signal beam $\mathrm{P}_{i}$, keeping $\mathrm{S} 1$ closed. The radio frequency driving $\mathrm{AO} 2$ is dithered $\pm 2 \mathrm{MHz}$ at a rate of $110 \mathrm{~Hz}$, and a standard phasesensitive detection and servo arrangement locks the offset frequency of the pumping beam to the transition. Hence the frequency of the signal beam is locked and detuned 73 $\mathrm{MHz}$ above the atomic transition frequency $\left(F=2, m_{F}=2\right.$ $\rightarrow F=3, m_{F}=3$ ).

The fluorescence from a monitor beam derived from the unshifted optical pumping beam is used for alignment purposes. This beam is retroreflected back through the atomic beam with a corner-cube prism, and the image of both forward and backward beams is focused onto a photomultiplier tube $\left(\mathrm{I}_{m}\right)$. The absorption profile observed in fluorescence is our reference in setting the directions of the various laser beams perpendicular to that of the atomic beam.

The LO beam and the signal beam emerging from the cavity are mode matched and combined at the beam splitter BS3. The phase of the LO is controlled by a voltage applied to a piezoelectric ceramic (PZT3) on which the mirror M3 is mounted. The light leaving the cavity has its polarization converted from circular to linear with the quarter-wave plate Q3 before it is mixed with the LO beam at BS3.

Beam splitter BS3 is the input to a balanced homodyne detector ${ }^{29}$ formed by the photodiodes SD1 and SD2. The photocurrents are amplified to produce the signals $i_{1}$ and $i_{2}$ that are combined in a $180^{\circ}$ splitter-combiner (Mini-Circuits ZSCJ-2-1). The relative noise level $\Phi(\phi, \Omega)$ of the output of the splitter is displayed in a spectrum analyzer (HP 8558B) and recorded using a digital storage oscilloscope (LeCroy 9400).

Two different sets of apertures and cavities are used in the experiments reported here, which we designate as configura- tions $(\mathbf{a}, \mathbf{b})$. For case a, the atomic beam is formed by a 0.5 $\mathrm{mm}$ aperture in the source oven and by a $0.5-\mathrm{mm}$ aperture located $280 \mathrm{~mm}$ downstream from the oven and $15 \mathrm{~mm}$ upstream from the cavity waist. The maximum absorption $\alpha_{m} l$ for this configuration is $\alpha_{m} l=0.3$. In the more recent arrangement $\mathbf{b}$ the atomic medium is formed by three 0.5$\mathrm{mm}$ apertures in the source oven lying along a line perpendicular to the plane of Fig. 4 and by a single $0.3-\mathrm{mm}$ aperture located $280 \mathrm{~mm}$ downstream from the oven and $15 \mathrm{~mm}$ upstream from the cavity waist. The maximum absorption measured for this configuration is $\alpha_{m} l=0.6$.

In configuration a the cavity is formed by a pair of mirrors with radius of curvature $R_{1} \mathrm{a}=100 \mathrm{~cm}$ and $R_{2}{ }^{\mathrm{a}}=50 \mathrm{~cm}$ separated by $1.2 \mathrm{~mm}$. The transmission coefficients of the mirrors are $T_{1}^{\mathrm{a}}=2 \times 10^{-4}$ and $T_{2}^{\mathrm{a}}=3 \times 10^{-3}$. The cavity finesse is $F^{\mathrm{a}}=1200 \pm 100$ with resonant cavity transmission of $T^{\mathrm{a}}=0.03$ and normalized decay rate of $\mu^{\mathrm{a}}=5.4 \pm 0.4$. Cavity $\mathbf{b}$ is formed by a pair of mirrors with radius of curvature $R_{1}{ }^{\mathrm{b}}=100 \mathrm{~cm}$ and $R_{2}{ }^{\mathrm{b}}=100 \mathrm{~cm}$ separated by $0.83 \mathrm{~mm}$. The transmission coefficients of the mirrors in this cavity are $T_{1}{ }^{b}=2 \times 10^{-4}$ and $T_{2}{ }^{b}=7.5 \times 10^{-3}$. The cavity finesse is $F^{\mathrm{b}}=660 \pm 30$, with resonant cavity transmission of $T^{\mathrm{b}}=$ 0.045 and normalized decay rate of $\mu^{\mathbf{b}}=13.5 \pm 0.5$. No active stabilization is employed for either cavity in these experiments; the time to drift across the resonance is longer than 30 sec. Cavity tuning is accomplished by manually varying the voltage applied to piezoelectric transducers (PZT1, PZT2) on which the cavity mirrors are mounted. The cavity waist $w_{0}$ for both geometries $(\mathbf{a}, \mathbf{b})$ is approximately $w_{0}=62 \mu \mathrm{m}$. The mixed Fresnel numbers $(F \#=$ $\pi w_{0}^{2} / \lambda l$, with $\lambda$ the wavelength of the radiation and $l$ the length of the atomic medium $)^{30}$ of the configurations are $F \#^{\mathrm{a}}=41$ and $F \#^{\mathrm{b}}=68$.

As indicated in Fig. 4, the interferometer and interaction region are in an evacuated region $\left(\approx 5 \times 10^{-7}\right.$ Torr $)$ provided by a diffusion pump. Cold traps around the cavity significantly reduce the background sodium vapor. The light transmitted through M2 travels approximately $2 \mathrm{~cm}$ inside the vacuum chamber before exiting through an antireflection-coated window.

Laser 1 is pumped by a Spectra-Physics 171-09 argon-ion laser operating on a single line at $514 \mathrm{~nm}$. The rms linewidth of the dye laser is $500 \mathrm{kHz}$. The rms intensity noise is less than $3 \%$ with a large contribution coming at 360 $\mathrm{Hz}$ traceable to the ion laser. An intracavity aperture is employed in the ion laser to ensure that only a single transverse mode excites the dye laser. In this form, the spectrum of dye-laser amplitude noise consists of a shot-noise level and coherent lines at multiples of the $83-\mathrm{MHz}$ ion-laser longitudinal mode spacing, instead of the dense forest of lines present when the aperture of the ion laser is wide open. That the laser 1 (and thus the LO for the detection of squeezing) is indeed at the vacuum level is confirmed by a comparison of the noise levels observed when the two photocurrents $i_{1}$ and $i_{2}$ are combined first with a $0^{\circ}$ and then with a $180^{\circ}$ phase shift (for a detailed discussion of this point see the paper by Wu et al. ${ }^{31}$ in this issue). We find that, in the wide regions between the coherent lines, the LO fluctuations are within $\pm 1 \%$ of the vacuum level over the spectral range of interest in the current experiment.

The photodiodes (SD1, SD2) employed in the balanced detector are EGG-FFD-060 with the glass windows removed 
and with the reflection from each diode surface collected and refocused onto the photodiode, resulting in a quantum efficiency $\alpha=0.85 \pm 0.04$. We operate the diodes with a bias voltage of $85 \mathrm{~V}$. The low-frequency components of photocurrents are dc coupled using an inductor of $0.5 \mu \mathrm{H}$ in series with a $100-\Omega$ resistor to provide a direct measure of the LO power. The high-frequency components are ac coupled and amplified $33 \mathrm{~dB}$ by a GPD201 amplifier from Avantek. The diodes and amplifiers are mounted on an etched board to reduce rf pick up. Over the range $100-300 \mathrm{MHz}$ the shot noise associated with the $2.5-\mathrm{mA}$ dc photocurrent produced by the LO exceeds the amplifier noise level by greater than 5 dB. The two detectors are matched so that, with the $180^{\circ}$ phase shift employed in the squeezing measurements, any excess $\mathrm{LO}$ noise is reduced by more than $12 \mathrm{~dB}$ in the range between $240-320 \mathrm{MHz}$ and $22 \mathrm{~dB}$ in the range between 80 $160 \mathrm{MHz}$.

To measure the intensity of the transmitted beam from M2 simultaneously with the detection of squeezing, we apply a $0.5 \%$ modulation at $30 \mathrm{kHz}$ to the intensity of the signal beam, using EO1. The dc output of one of the photodiodes (SD1, SD2) is sent to a lock-in amplifier whose output displays the interference fringe between signal and LO beams as the LO phase is scanned. The size of the fringe (typically a $0.01 \%$ modulation of the total photocurrent) is proportional to the field leaving the cavity and when calibrated gives the power of the squeezed beam.

\section{ALIGNMENT OF THE APPARATUS AND STEADY-STATE CHARACTERISTICS}

Since our optical cavity is fixed without the possibility of tilting or displacement, the alignment of the apertures that form the atomic beam is critical. We need to be sure that the atomic beam crosses at the center of the interferometer and is as close to perpendicular to the optic axis of the cavity as possible to avoid any Doppler contribution. We first align the signal beam to excite the cavity in the TEM $\mathrm{TO}_{00}$ mode and employ an autocollimator to bring the direction of the collimating apertures perpendicular to the cavity axis. We then load the oven with $25 \mathrm{~cm}^{3}$ of metallic sodium and close the vacuum chamber. Next comes the alignment of the laser beams perpendicular to the atomic beam. The monitor beam is retroreflected with a corner cube, and the image of both beams is focused onto a photomultiplier tube $I_{m}$. We steer the monitor beam while scanning the laser frequency $\pm 100 \mathrm{MHz}$ from atomic line center and examine the fluorescence from the incoming and outgoing beams. When both signals are on resonance at the same frequency, we know that the monitor beam is perpendicular to the atomic beam. The fluorescence from the monitor beam then serves as the reference to align the optical pumping beam. To measure the perpendicularity of the signal beam with respect to the atomic-beam direction (which unfortunately is not adjustable when the system is evacuated), we tune the cavity off resonance and increase the signal-beam intensity to a high level (recall that the transmission of $\mathrm{M} 1$ is $T_{1}=2 \times$ $10^{-4}$ and that the cavity free spectral range is approximately $150 \mathrm{GHz}$ ). In this way we can observe the fluorescence signal from the atomic beam inside the cavity, without having to cope with the bistability that occurs near the cavity resonance and can make a comparison with the signals com- ing from the monitor beam and the optical pumping beam. For the experiments reported here, the alignment of all beams is such that the centers of the fluorescent profiles coincide to well within $\pm 3 \mathrm{MHz}$ as the frequency of the laser is scanned.

Two methods are used to measure the beating efficiency $\eta$ (Ref. 32) between the LO and the output signal beam from the cavity (M1, M2). In the first case we measure the interference fringe between the LO and the signal beam for an empty cavity tuned to resonance as the LO phase is scanned. The measurement is independent of diode calibration since only relative powers are required and can be performed in situ using the dc outputs of the photodiodes SD1 and SD2. A second technique is used to check consistency. We shift the signal-beam frequency with the acousto-optic modulator AO1 and then measure the coherent heterodyne signal between the LO and the signal beam from (M1, M2). Although the acousto-optic modulator displaces the frequency-shifted signal beam, the cavity mode for optimal transmission fixes the direction of the input beam. Thus it is possible to retrace with the frequency-shifted beam the actual path of the unshifted output beam. The absolute output power of the signal beam and the ratio of the shot-noise level (produced by the LO alone) to the size of the beat note (with offset signal and LO present) are needed to calculate the heterodyne efficiency. Both measurements give consistent results.

Knowledge of the cooperativity $C$ during the experiments is important since it gives, when combined with other parameters of the system, the coupling rate $g \sqrt{N}=\gamma \sqrt{\mu C}$ between the atoms and the cavity field that is of fundamental importance for the generation of squeezing in the regime that we explore. Our technique is to measure the ratio $R$ of input switching points $\left(Y_{1}, Y_{2}\right)$ for absorptive optical bistability (Fig. 5). This ratio is proportional to an effective cooperativity $C_{\text {eff }}, 33$ which for the case of no Doppler broadening is just C. ${ }^{34}$ Previous experiments performed by ourselves show good absolute agreement between the values of

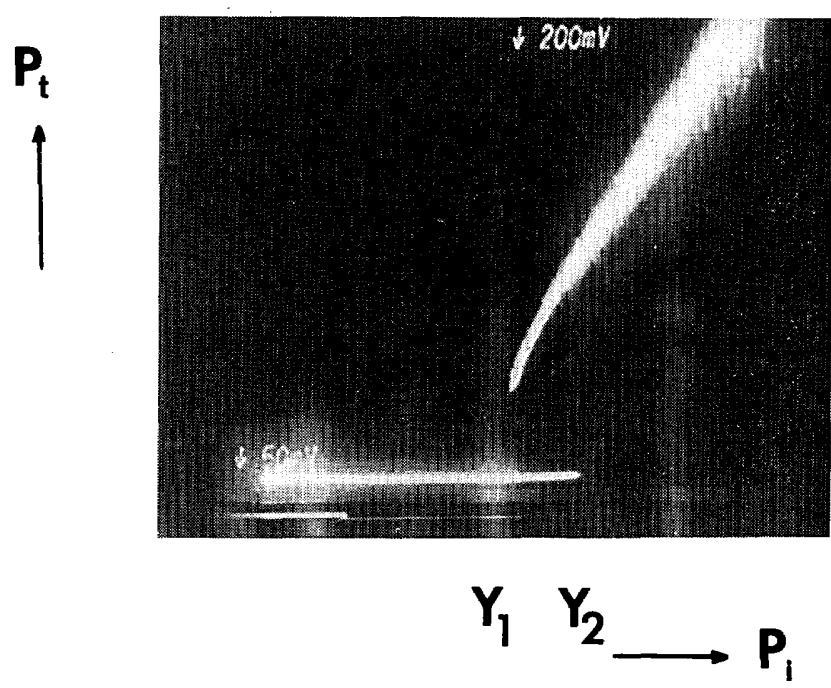

Fig. 5. Oscilloscope trace $\left(P_{i}\right.$ versus $\left.P_{t}\right)$ of the steady-state characteristics of absorptive optical bistability for case $b$. From the ratio $R$ of input switching intensities $Y_{1}, Y_{2}\left(R=Y_{2} / Y_{1}\right)$ in traces such as this one, we determine the cooperativity parameter $C$. In this trace the input switching powers corresponding to $\left(Y_{1}\right.$ and $\left.Y_{2}\right)$ are $P_{1}=87$ $\pm 10 \mu \mathrm{W}$ and $P_{2}=103 \pm 12 \mu \mathrm{W}$, respectively. 

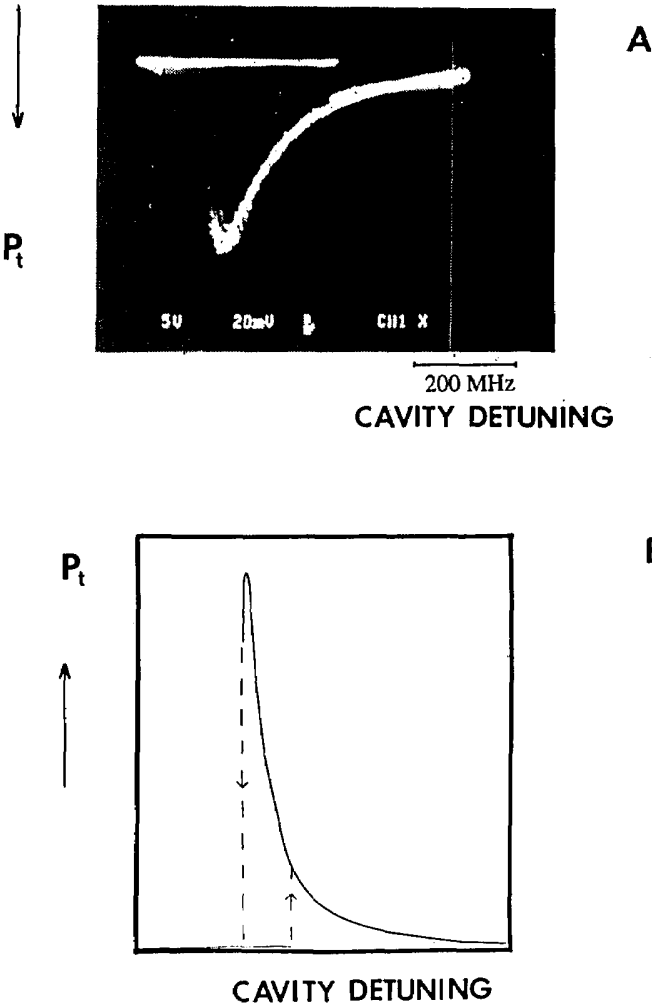

Fig. 6. A, Oscilloscope trace of the transmitted intensity $P_{t}$ as a function of cavity detuning $\theta$ in the presence of atomic detuning $\Delta$. The peak transmission from the cavity is $2.3 \pm 0.3 \mu \mathrm{W}$ and a scale for the cavity detuning is given. Note that the hysteresis associated with the scanning of the PZT was not corrected and accounts for a broadening of about $12 \mathrm{MHz}$, which is a small fraction of the detuning range between turning points shown in the figure. $\mathrm{B}$, Theoretical prediction for the transmitted intensity $P_{t}$ as a function of cavity detuning $\theta$ with atomic detuning $\Delta$.

$C$ calculated from independent measurements of the atomic absorption $\alpha l$ and of the cavity losses and the values of $C$ inferred from the ratios of incident switching intensities $\left(Y_{1}, Y_{2}\right){ }^{18}$ To record the input-output characteristics $\left(\dot{P}_{i}\right.$ versus $P_{t}$ ), shutter $\mathrm{S} 2$ is closed, $\mathrm{S} 1$ is opened, and the input intensity is scanned at $50 \mathrm{~Hz}$ by EO2. The LO beam is blocked with the shutter $\mathrm{S} 3$ and the mirror $\mathrm{M}_{t}$ inserted in front of SD1 to direct the output from the cavity onto a calibrated photomultiplier tube $\mathrm{P}_{t}$. The cavity is manually held on resonance by applying a voltage to PZT2; the point-zero atomic detuning $(\Delta=0)$ is identified by an independence of the input-output characteristics on the sign of the cavity detuning. The ratio $R$ is measured at several intracavity densities, and the optical pumping fluorescence is calibrated against $R$ to provide a continuous monitor during the squeezing measurements.

Next we explore the output characteristics as a function of cavity detuning when the atoms are excited off line center with the input intensity kept constant. We do so to record the deterministic characteristics of the system in the presence of squeezing. The cavity detuning is scanned up and down through the cavity resonance by ramping the voltage to PZT1. Figure 6 shows the resulting line shape for configuration a. The squeezing that we report occurs along the lower branch in this photograph. Figure $6 \mathrm{~B}$ is a theoretical prediction based on the steady-state equation from the work of Drummond..$^{34}$

\section{OBSERVATION OF SQUEEZING}

The parameter space to be searched in our investigation of squeezed-state generation is large, with the analysis frequency $\Omega$, the atomic detuning $\Delta$ (measured in units of $\gamma_{\perp}$, where we take the radiative value $\gamma_{\perp} / 2 \pi=5 \mathrm{MHz}$ for the sodium transition that we study), the cavity detuning $\theta$, the incident field $y$, and the atomic cooperativity parameter $C$ as externally controlled variables. In this space the degree of squeezing is a fairly smooth function of the system parameters. To limit the paths on which we can wander, we proceed as follows: first we fix the atomic detuning $\Delta$, cooperativity $C$, and input driving power $P_{i}$. The spectrum analyzer is set to scan over a wide frequency range of about $50 \mathrm{MHz}$. The video output of the spectrum analyzer is low-pass filtered and recorded in a LeCroy 9400 digital storage oscilloscope. While the LO phase is continuously scanned, we manually tune the cavity length. Our search is of course for phasesensitive noise reductions below the vacuum level. Figure 7 is characteristic of traces recorded in this search; note that noise reductions below the vacuum level are observed over quite broad regions in analysis of frequency $\Omega$. We search in segments over broad regions of analyzing frequency $\Omega$ and modify in steps the input intensity and atomic detuning to optimize the noise reductions, with the cavity detunings continuously optimized manually. When a region in parameter space with good squeezing is identified, we stop the scan of the spectrum analyzer and examine the fluctuations in a narrow bandwidth at the optimum $\Omega$.

An example of our observations of noise reductions below the vacuum level is given in Fig. 8. The figure displays the level of photocurrent fluctuations $\Phi(\phi, \Omega)$ on a logarithmic scale at fixed analysis frequency (obtained following the procedure described above) $\Omega / 2 \pi=124 \mathrm{MHz}$ versus $\mathrm{LO}$

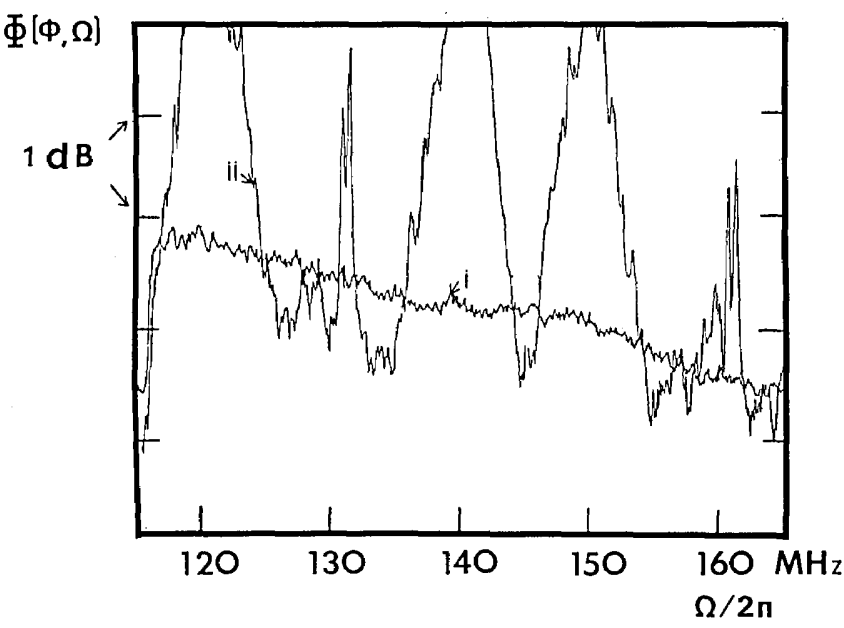

Fig. 7. Relative level $\Phi(\phi, \Omega)$ of fluctuations of the difference photocurrent $\left(i_{1}-i_{2}\right)$ on a logarithmic scale as a function of analysis frequency $\Omega / 2 \pi$. The LO phase $\phi$ is being swept at a rate somewhat higher than the rate at which $\Omega$ is varied. The data are for configuration b with $C=20 \pm 5, \Delta=7 \pm 1$. Trace (i) Signal input blocked to define the vacuum level (the sloping character of this trace is due to the high-frequency falloff of the response of the photodiodeamplifier configuration). Trace (ii) Phase-sensitive fluctuations with signal beam present drop below the vacuum level. Traces such as this one over several ranges of $\Omega$ help us to search the parameter anner and to find the region of hest squeezing. The time taken for this trace is $0.2 \mathrm{sec}$; the sharp features near 130 and $160 \mathrm{MHz}$ are generated by the flyback of the piezoelectric transducer. 


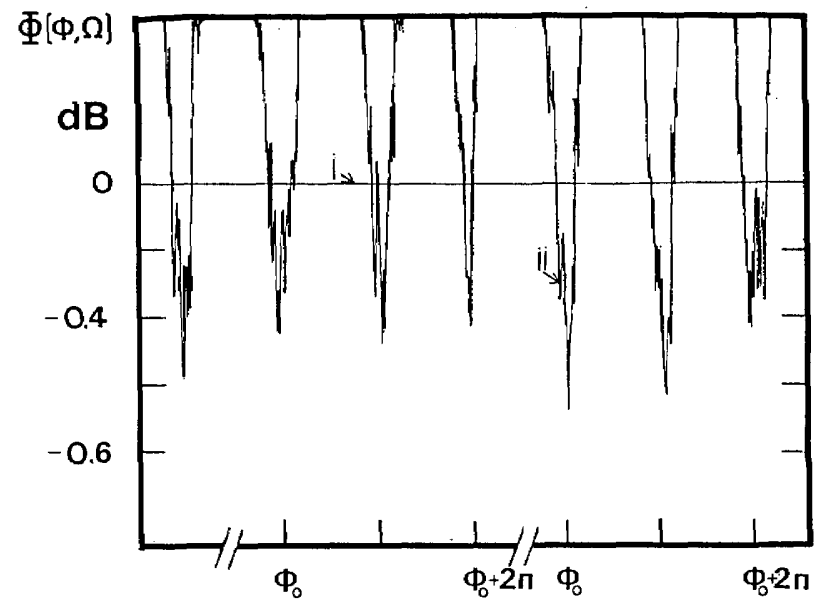

Fig. 8. Relative noise level $\Phi(\phi, \Omega)$ of fluctuations of the difference photocurrent $\left(i_{1}-i_{2}\right)$ versus $L O$ phase $\phi$ at fixed analysis frequeney $\Omega / 2 \pi=124 \mathrm{MHz}$ and bandwidth $B=300 \mathrm{kHz}$ (postdetection video bandwidth, $\Delta f=106 \mathrm{~Hz}$ ) for configuration a. Trace (i) Signal input blocked to define vacuum level (multiple trace average). Trace (ii) Phase-sensitive fluctuations with signal beam present drop below the vacuium level. Operating conditions $C=28 \pm 3, \mu=5.4 \pm 0.4, \Delta$ $=7.0 \pm 1.0, \theta=-2 \pm 1, x=1$ (within a factor of 3 ), $P_{i}=77 \pm 10 \mu \mathrm{W}$. The time taken for the trace is $0.5 \mathrm{sec}$. The signal was not corrected for the electronic noise contribution.

phase $\phi$ for $C=28 \pm 3$, which for $\mu=5.4 \pm 0.4$ corresponds to a vacuum-field Rabi splitting $g \sqrt{N}=123 \pm 10 \mathrm{MHz}$. Trace (i) is the vacuum-plus-amplifier noise level obtained by either blocking the signal beam, blocking the atomic beams, or detuning the cavity. Trace (ii) is recorded with the signal beam present and exhibits noise above and below the vacuum level as the $\mathrm{LO}$ phase is varied. Note that the periodicity of the reductions with LO phase $\phi$ is $\pi$ rather than the $2 \pi$ periodicity of intensity fringes in the system. The atomic detuning for this trace is $\Delta=7 \pm 1$, the cavity detuning $\theta=-2 \pm 1$, the intracavity field $x$ is of the order of 1 (within a factor of 3 ), and the power $P_{i}$ incident upon M1 equals $P_{i}=77 \pm 10 \mu \mathrm{W}$.

As the squeezed light propagates out of the cavity toward the photodetectors, the degree of squeezing is degraded by a variety of mechanisms (see Ref. 31 in this issue for a detailed discussion). The first one is the escape efficiency $\rho$ from the cavity itself, where $\rho$ is the ratio of the measured finesse to the finesse inferred from the value of $T_{2}$, assuming $T_{1}$ to be zero (the ideal single-ended cavity). The optical elements that the squeezed light encounters introduce a propagation loss factor of $\left(1-T_{0}\right)$. At the output of the Mach-Zehnder interferometer at which the signal mixes with the LO, a heterodyne efficiency $\eta$ accounts for imperfect matching of the two beams; finally, in the photodetectors one can lose squeezing through detector quantum efficiency $\alpha$. By measuring all the propagation losses, one can relate the observed noise reduction $R_{-}$to the spectrum of squeezing $S_{-}$(Refs. 3,35 , and 36):

$$
R_{-}(\Omega)=1+\rho T_{0} \alpha \eta^{2} S_{-}(\Omega) \text {. }
$$

The noise enhancement $R_{+}(\Omega)$ is likewise related to $S_{+}(\Omega)$ through Eq. (4) by substituting the subscript - by + . As displayed in Fig. $8, R_{ \pm}$is given simply by $R_{ \pm}=10^{\Phi / 10}$ once the electronic-noise correction to $\Phi$ has been made. By separate measurements of each of the quantities, we find that $\left(\rho, T_{0}, \alpha\right.$, $\eta)=(0.79 \pm 0.06,0.97,0.85 \pm 0.04,0.93 \pm 0.07)$ for configuration b. We thus can infer $S_{-}$from measurement of $R_{-}$.

While doing the first experiments in configuration $\mathbf{b}$, we encountered a problem with the atomic beams of sodium. From measurements of the fluorescence for weak excitation, we found the absorption linewidth broadened from $13 \mathrm{MHz}$ (its normal transit broadened value inferred from previous measurements ${ }^{17,18}$ to $26 \mathrm{MHz}$ indicating a Doppler contribution that might be degrading the squeezing. However, we believe that, for the off-resonance detuning of $75 \mathrm{MHz}$ used in these experiments, the effect of this Doppler broadening is not very important either to the degree of squeezing or to the frequency $\Omega$ at which it occurs. Some preliminary theoretical averages over different atomic detunings indicate a small effect on the amount of squeezing predicted for the parameters in the experiment. To infer $C$ in the Dopplerbroadened case, we first determine $C_{\text {eff }}$ (Ref. 33 ) from our measured ratios of input switching points $R$ against the optical pumping fluorescence $I_{\mathrm{op}}$. For this conversion of $R$ to $C_{\text {eff }}$ one requires a table of turning points generated from a state equation in absorptive bistability that includes the observed degree of inhomogeneous broadening. ${ }^{34}$ The value of $C$ then follows since for the degree of Doppler broadening in our work $C \approx 2 C_{\text {eff. }}$. The values of $C$ quoted for Figs. 8-10 are obtained in this fashion.

Figure 9 shows the dependence of the noise voltage $V(\phi)$ on LO phase $\phi$ for the signal produced in the lower branch of optical bistability for $C=45 \pm 6$ and $\Delta=-14.6$. After the correction for the amplifier noise contribution is made, the observed noise reduction in this trace corresponds to a $26 \%$ reduction below the vacuum level $\left(R_{-}=0.74\right)$. By taking into account the losses with Eq. (4), we find that the product of the quadrature uncertainties is $M=\left(1+S_{+}\right)\left(1+S_{-}\right)=$ $1.3 \pm 0.15$. The field state is thus not a minimum-uncertain-

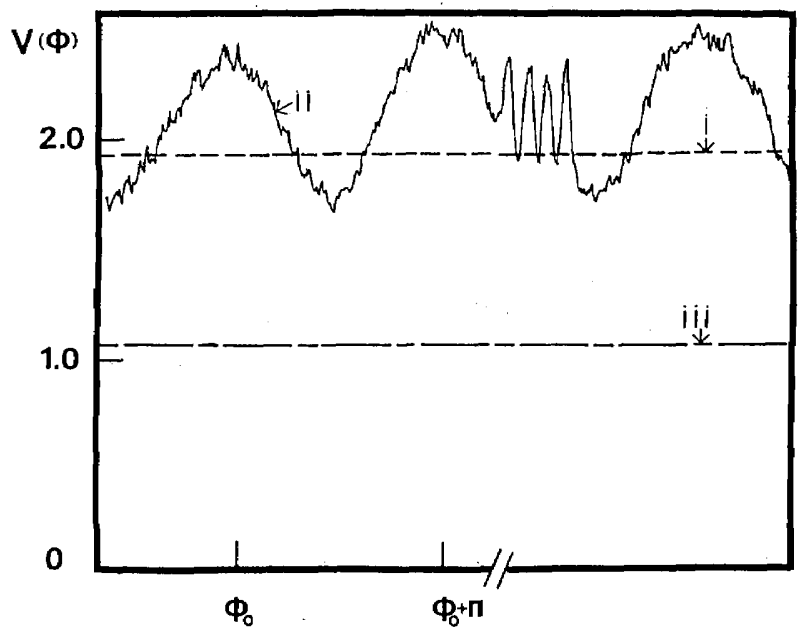

LOCAL OSCILLATOR PHASE

Fig. 9. Dependence of rms noise voltage $V(\phi)$ on LO phase $\phi$ at fixed analysis frequency $\Omega / 2 \pi=280 \mathrm{MHz}$ and bandwidth of $300 \mathrm{kHz}$ (postdetection video bandwidth, $159 \mathrm{~Hz}$ ) for case b. $C=45 \pm 6, \Delta=$ $-14.6 \pm 0.2$. Trace (i) is the vacuum-plus-amplifier noise, trace (ii) is the squeezed beam signal, and trace (iii) is the amplifier noise level alone obtained with no light on the photodiodes. The sharp feature at the right side of the trace is due to the flyback of the piezoelectric used to scan the phase of the LO. Note that the ordinate is a linear scale in noise voltage. The time taken for the trace is $0.2 \mathrm{sec}$. 


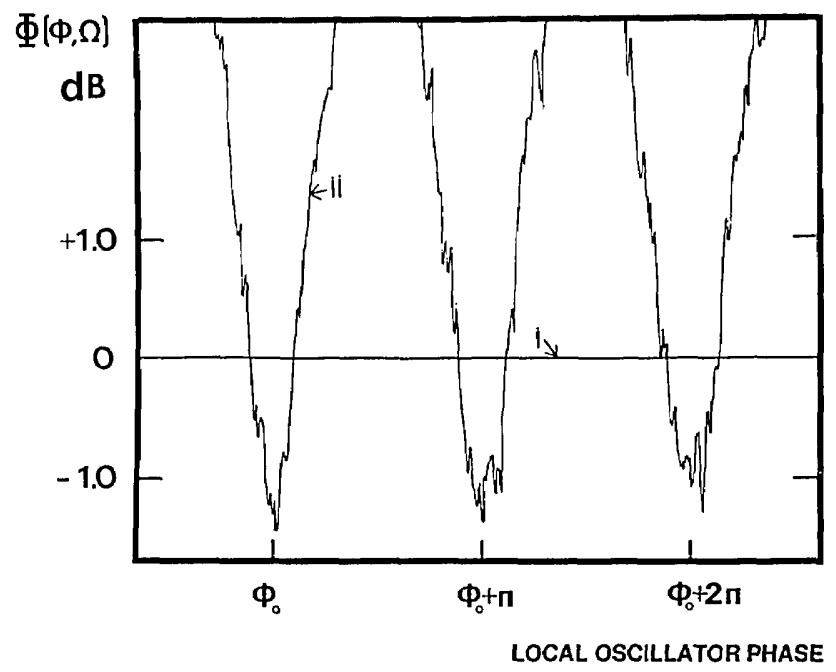

Fig. 10. Relative noise level $\Phi(\phi, \Omega)$ of fluctuations of the difference photocurrent $\left(i_{1}-i_{2}\right)$ versus LO phase $\phi$ at fixed analysis frequency $\Omega / 2 \pi=280 \mathrm{MHz}$ and bandwidth of $300 \mathrm{kHz}$ (postdetection video bandwidth, $159 \mathrm{~Hz}$ ) for case $\mathbf{b}$. Trace (i) Signal input blocked to define vacuum level. Trace (ii) Phase-sensitive fluctuations with signal beam present drop below the vacuum level. Operating conditions $C=52 \pm 8, \mu=13.5 \pm 0.5, \Delta=-14.6 \pm 0.2, x=25 \pm$ $5, P_{i}=400 \pm 100 \mu \mathrm{W}$. The time taken for the trace is 0.2 sec.

ty state for which $M=1$. This observation is in agreement with our theoretical calculations, which indicate that the state of the field obtained with this squeezing mechanism should not be a minimum-uncertainty state and, in fact, should depart more and more from the situation $M=1$ as the degree of squeezing gets larger. In Fig. 9, trace (i) is the vacuum-plus-amplifier noise level, trace (ii) is the signal with the squeezed beam present, and trace (iii) is the amplifier noise level obtained with both $\mathrm{LO}$ and signal beams blocked.

Figure 10 represents a trace taken at $\Omega / 2 \pi=280 \mathrm{MHz}$ (optimized as described above), $\Delta=-14.6, x=25 \pm 5$, and $C$ $=52 \pm 8$, giving a coupling of $g \sqrt{N}=265 \pm 30 \mathrm{MHz}$. From traces such as the one shown in Fig. 10, we construct histograms of noise levels around the minima. Figure 11 is generated in this fashion from data at the same parameters as in Fig. 10. From this figure we arrive at a number of -1.0 $\pm 0.13 \mathrm{~dB}$ (upper scale) for the recorded noise reduction averaged over the entire distribution, which becomes -1.55 $\pm 0.19 \mathrm{~dB}$ (lower scale) after correction is made for the nonzero noise level of the amplifier (again, this is the average of the histogram). This number represents a level of fluctuations $R_{-}=0.70 \pm 0.04$, which is $30 \%$ below the level set by the vacuum state of the field at the signal port of the balanced homodyne detector. For this case the measured $R_{-}$corresponds to $S_{-}=(-0.53 \pm 0.09)$, which is somewhat smaller than the theoretical prediction of -0.78 [Fig. 12, trace (ii)] based on a plane-wave model with all parameters optimized. 9,26

To investigate the role of Doppler broadening in our first experiments in the configuration $b$, we repeated the experiments with this Doppler broadening largely eliminated. (A faulty seal in the oven along a direction parallel to the cavity axis was responsible for an effective increase in the transverse dimension of the source aperture.) Although the directly observed noise reductions were somewhat larg- er $(-1.2 \mathrm{~dB})$ because of a decreased contribution from the amplifier noise level, the results obtained in the new experiments agree quite well with those quoted above. In particular, for operation in a parameter regime comparable with that of Fig. 10, we observe noise reductions of $R_{-} \approx 0.70$ (after correction for nonzero amplifier noise) for an analysis frequency of $280 \mathrm{MHz}$, as shown in Fig. 12.

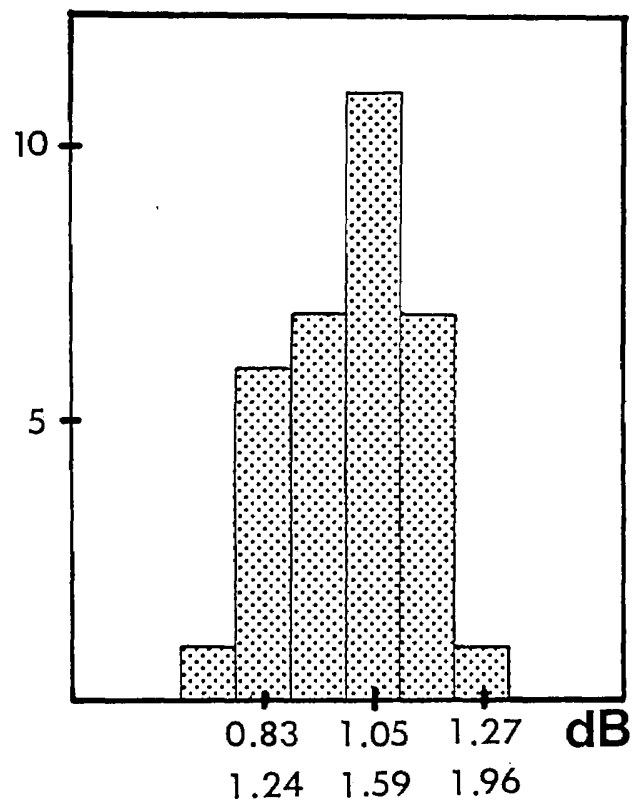

Fig. 11. Histogram of frequency of occurrence of a given noise level around the minima of $\Phi(\phi, \Omega)$ obtained from many traces with the same parameters as in Fig. 10. The upper abcissa is the actual recorded noise reduction below the vacuum noise level, whereas the lower abcissa is the noise reduction after correction is made for the nonzero noise level of the amplifier. The asymmetry of the distribution presumably arises because there is some largest noise reduction produced by the system. For constant control parameters, the statistical fluctuations in photocurrent would give rise to a symmetric distribution, with $\mathrm{HWHM}$ indicated by the right-hand side of the figure. Since the control parameters are not constant, optimum squeezing is not recorded at each noise minimum, thereby skewing the distribution on the left-hand side.

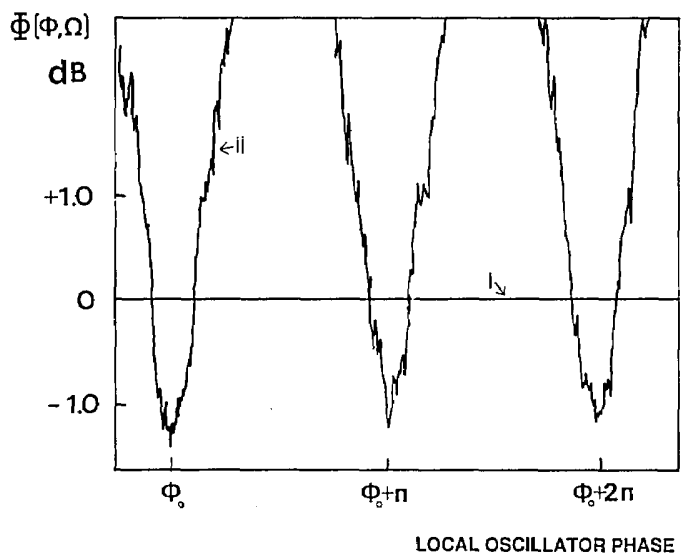

Fig. 12. Relative noise level $\Phi(\phi, \Omega)$ of fluctuations of the difference photocurrent $\left(i_{1}-i_{2}\right)$ versus LO phase $\phi$ at fixed analysis frequency $\Omega / 2 \pi=280 \mathrm{MHz}$ and bandwidth of $300 \mathrm{kHz}$ (postdetection video bandwidth, $159 \mathrm{~Hz}$ ), with the source of Doppler broadening corrected. Trace (i) Signal input blocked to define vacuum level. Trace (ii) Phase-sensitive fluctuations with signal beam present drop below the vacuum level for $C=52 \pm 10, \mu=13.5 \pm 0.5$, and $\Delta=-14.6 \pm 0.2$. 
Preliminary explorations of the dependence of $S_{-}(\Omega)$ on analysis frequency $\Omega$ show large regions of squeezing that extend over a range roughly of the order of the cavity linewidth $\kappa$, which in our experiments (case b) is $\kappa / 2 \pi \sim 135$ $\mathrm{MHz}$. Close to the carrier frequency $(\Omega \sim 0)$, there are large degrees of phase-sensitive noise but no reductions below the vacuum level. This study is carried out for configuration b, based on traces similar to the one shown in Fig. 7 . These results are in qualitative agreement with theoretical predictions such as the ones shown in Fig. 2.

In quantitative terms, note that for the two cavities studied ( $\mu^{\mathrm{a}}=5.4$ and $\left.\mu^{\mathrm{b}}=13.5\right)$, there is a good correspondence between the observed frequencies of optimum noise reduction and the simple prediction $\Omega_{0}=g \sqrt{N}=\gamma \sqrt{\mu C}$ suggested by Fig. 1. In particular, for configuration a, we find that the frequencies of optimum squeezing are $(\Omega / 2 \pi)_{\exp }=124$ $\mathrm{MHz}$ and $\left(\Omega_{0} / 2 \pi\right)=123 \pm 10 \mathrm{MHz}$ for $C=28 \pm 3$. In configuration $\mathbf{b}$, we find optimum frequencies $(\Omega / 2 \pi)_{\exp }=$ $280 \mathrm{MHz}$ and $\left(\Omega_{0} / 2 \pi\right)=265 \pm 30 \mathrm{MHz}$ for $C=52 \pm 8$.

Although we did not explore in great detail the upper branch of the bistable transmission characteristic (Fig. 5), we found no evidence for noise reductions below the vacuum level but do see phase-sensitive noise across the spectrum.

\section{CONCLUSIONS}

We have identified a new regime for squeezed-state generation associated with the coupling of a collection of two-level atoms to an optical cavity. In terms of the deterministic characteristics of the system the squeezing occurs along the lower branch in the mixed regime of absorptive and dispersive optical bistability. The physical process responsible for the squeezing is a coupling-induced mode splitting in the eigenvalue spectrum of the system, which for weak fields and zero detunings is just the vacuum-field Rabi splitting $g \sqrt{N}$. Noise reductions of $30 \%$ relative to the vacuum noise level have been observed. The large region in radio frequency $\Omega$ over which the squeezing extends is one of the largest observed in squeezed-state experiments.

While the degree of squeezing observed in our experiments is somewhat less than that predicted by our theory, a detailed comparison of experimental results with theoretical predictions is hampered at present by large uncertainties in the experimental parameters and by the inefficiency with which we search in a rather large parameter space. One cause for concern of a more fundamental nature is the fact that the theory is carried out for a traveling-wave interferometer with plane waves, whereas the experiments are conducted in a standing-wave cavity with a Gaussiantransverse profile. While we are reasonably confident that the deterministic physics is adequately described by a single-transverse-mode theory, ${ }^{18}$ the precise role of a nonuniform cavity mode in altering the quantum fluctuations is yet to be understood, although our group has carried out a full quantum treatment in the good-cavity limit. ${ }^{37}$ Other mechanisms that also need consideration in the model are the small residual broadenings present in the system (transient and Doppler) that could contribute to reducing the amount of squeezing attainable.

We do stress that the frequency $\Omega$ at which optimum squeezing occurs is in quantitative agreement with the pre- diction of our theoretical analysis. This fact leads to some optimism regarding the reliability of the theory as applied to our experiment. If the current discrepancies between theory and experiment about the degree of squeezing can be resolved, then one might believe that the very large degrees of squeezing ( $S_{-}<-0.92$ or a factor of 12.5 squeezing) indicated in trace (iii) of Fig. 1 could be reached with suitable modifications of our apparatus.

Quite apart from the particular system of two-level atoms in a cavity, we suggest that the mechanism that we have described might be more generally useful in a variety of circumstances for the generation of squeezed states of light. For extremely small integrated devices for which the cavity damping rate approaches the extremely fast relaxation rates of the nonlinear medium, a normal mode structure similar to the one that we described could arise, although our analysis is strictly valid only in the case of a high-finesse cavity. This structure offers the possibility of squeezed-state generation in a cavity not by the simple compounding of the nonlinearity of the medium in multiple passes but rather by employing the mode splitting of the composite entity of cavity plus medium.

\section{ACKNOWLEDGMENTS}

This research was supported by the Venture Research Unit of British Petroleum, North America, the U.S. Office of Naval Research, and the National Science Foundation. L. A. Orozco acknowledges support from an IBM graduate fellowship.

\section{REFERENCES}

1. B. Yurke, Phys. Rev. A. 29, 408 (1984).

2. R. E. Slusher, L. W. Hollberg, B. Yurke, J. C. Mertz, and J. F. Valley, Phys. Rev. Lett. 55, 2409 (1985); R. E. Slusher, B. Yurke, P. Grangier, A. La Porta, D. F. Walls, and M. Reid, J. Opt. Soc. Am. B 4, 1453 (1987).

3. L.-A. Wu, H. J. Kimble, J. O. Hall, and H. Wu, Phys. Rev. Lett. 57, 2520 (1986); L.-A. Wu, M. Xiao, and H. J. Kimble, J. Opt. Soc. Am. B 4, 1465 (1987).

4. L. A. Lugiato and G. Strini, Opt. Commun. 41, 67 (1982).

5. M. D. Reid and D. F. Walls, Phys. Rev. A 32, 396 (1985); 34, 4929 (1986).

6. D. A. Holm and M. Sargent III, Phys. Rev. A 35, 2150 (1987).

7. H. J. Kimble, M. G. Raizen, L. A. Orozco, Min Xiao, and T. L. Boyd, in Fundamentals of Quantum Optics II, F. Ehlotzky, ed. (Springer Verlag, Berlin, 1987).

8. M. G. Raizen, L. A. Orozco, M. Xiao, T. L. Boyd, and H. J. Kimble, Phys. Rev. Lett. 59, 198 (1987).

9. H.-I. Yoo and J. H. Eberly, Phys. Rep. 118(5), 239 (1985).

10. G. S. Agarwal, J. Opt. Soc. Am. B 2, 480 (1985).

11. H. J. Carmichael, Phys. Rev. A 33, 3262 (1985).

12. J. Seke, J. Opt. Soc. Am. B 2, 1687 (1985); Phys. Rev. A 33, 739 (1986).

13. Y. Kaluzny, P. Goy, M. Gross, J. M. Raimond, and S. Haroche, Phys. Rev. Lett. 51, 1175 (1983).

14. D. Meschede, H. Walther, and G. Müller, Phys. Rev. Lett. 54, 551 (1985); G. Rempe, H. Walther, and N. Klein, Phys. Rev. Lett. 58, 353 (1987).

15. A. Heidmann, J. M. Raimond, and S. Reynaud, Phys. Rev. Lett. 54, 326 (1985); A. Heidmann, J. M. Raimond, S. Reynaud, and N. Zagury, Opt. Commun. 54, 54 (1985).

16. R. J. Brecha, L. A. Orozco, M. G. Raizen, Min Xiao, and H. J. Kimble, J. Opt. Soc. Am. B 3, P238 (1986).

17. A. T. Rosenberger, L. A. Orozco, and H. J. Kimble, Phys. Rev. A 28, 2569 (1983). 
18. L. A. Orozco, H. J. Kimble, and A. T. Rosenberger, Opt. Commun. 62, 54 (1987); Phys. Rev. A (to be published).

19. L. A. Lugiato, in Progress in Optics, E. Wolf, ed. (North-Holland, Amsterdam, 1984), Vol. 21, p. 69.

20. P. D. Drummond and D. F. Walls, J. Phys. A 13, 725 (1980).

21. P. D. Drummond and D. F. Walls, Phys. Rev. A 23, 2563 (1981).

22. H. J. Carmichael, D. F. Walls, P. D. Drummond, and S. S. Hassan, Phys. Rev. A 27, 3112 (1983).

23. H. J. Carmichael, Quantum Statistical Methods in Quantum Optics (Springer-Verlag, Berlin, to be published).

24. M. J. Collet and C. W. Gardiner, Phys. Rev. A 30, 1386 (1984); C. W. Gardiner and M. J. Collett, Phys. Rev. A 31, 3761 (1985).

25. M. J. Collet and D. F. Walls, Phys. Rev. A 32, 2887 (1985).

26. M. G. Raizen, L. A. Orozco, and H. J. Kimble, "Squeezed states in a coupled system" (submitted to Phys. Rev. A.)

27. M. D. Reid, A. Lane, and D. F. Walls, in Quantum Optics IV, J. D. Harvey and D. F. Walls, eds. (Springer-Verlag, Berlin, 1986), p. 31.

28. G. V. Varada, M. S. Kumar, and G. S. Agarwal, Opt. Commun. 62, 328 (1987).
29. H. P. Yuen and V. W. S. Chan, Opt. Lett. 8, 177 (1983); errata, Opt. Lett. 8, 345 (1983); B. L. Schumaker, Opt. Lett. 9, 189 (1984).

30. L. A. Lugiato and M. Milani, Z. Phys. B 50, 171 (1983).

31. L.-A. Wu, M. Xiao, and H. J. Kimble, J. Opt. Soc. Am. B 4, 1465 (1987).

32. L. Mandel and E. Wolf, J. Opt. Soc. Am. 65, 413 (1975).

33. H. J. Kimble, D. E. Grant, A. T. Rosenberger, and P. D. Drummond, in Laser Physics, J. D. Harvey and D. F. Walls, eds., Vol. 182 of Lecture Notes in Physics (Springer-Verlag, Berlin, 1983), p. 14.

34. P. D. Drummond, IEEE J. Quantum Electron. QE-17, 301 (1981); Department of Physics, University of Auckland, Private Bag, Auckland 1, New Zealand (personal communication).

35. L. Mandel, in Optics in Four Dimension-1980, M. A. Machado and L. M. Narducci, eds., AIP Conference Proceedings No. 65 (American Institute of Physics, New York, 1981).

36. J. H. Shapiro, IEEE J. Quantum Electron. QE-21, 237 (1985).

37. M. Xiao, H. J. Kimble, and H. J. Carmichael, Phys. Rev. A 35, 3832 (1987). 